\section{Up the cosmos}

\section{Hunting Down the Universe: The Missing Mass, Primordial Black Holes and Other Dark Matters}

by Michael Hawkins

Little, Brown: 1997. Pp. 278. £18.99. To be published in the United States by AddisonWesley on 1 October at $\$ 25$

\section{William H.Press}

Occasionally a book can be judged by its cover. This book's cover is black-on-black and barely decodable if you tip it at an angle to the light. You just know that this is going to be an outrageous book, and the author, the Edinburgh astronomer Michael Hawkins, does not disappoint.

He begins with a fierce attack on the likes of Steven Weinberg, Karl Popper and anyone else with the temerity to suggest that science is an objective search for an underlying reality. Addressing the suspicions of the most vehement critics of organized science, he states that, yes, "science is a social process beset by vested interests and power struggles, and scientific 'truth' is therefore determined by consensus, or even complicity". Strong stuff, this. Wittgenstein and Fred Hoyle also figure prominently, but one has a hard time keeping track of what side each is on. The general idea is that good theories - terrific theories even - get rejected by the "establishment" as they break too many rice bowls.

It is not too much of a surprise to learn that the author has a little theory of his own which, if true, is nothing less than "the most important cosmological breakthrough of the century". Is he pulling our leg, or is there something here to be taken seriously? Or a little of each?

Hawkins believes that small, primordial, omnipresent black holes, each about the size of a basketball and with a mass comparable to that of the planet Jupiter, comprise the cosmological "missing" mass. He believes there are enough such black holes to close the Universe and bring it into consistency with Guth's elegant inflationary model.

Virtually all astrophysicists view this hypothesis as highly improbable, for a variety of solid reasons. But it is not flatly contradicted by existing data. Moreover, the hypothesis does make predictions - quite remarkable predictions - that are subject to verification or disproof. As the hypothetical objects drift randomly across the line of sight between us and any distant quasar, their gravitational fields will momentarily focus the quasar's light in our direction. The result is that quasars should fluctuate in brightness.

Quasars do in fact fluctuate in brightness! All astrophysicists except Hawkins believe that these fluctuations are intrinsic to the quasar and are due to flarings of the unstable disc of gas that makes the quasar shine. Hawkins is pretty much alone in believing that the fluctuations are the proof of his theory, and that the "establishment", embodied in the Astronomer Royal Sir Martin Rees (an unlikely villain if ever there was one), is prejudiciallyblind to the truth. So this book is his appeal to that greater court of educated lay opinion.

It's a good yarn. But I think the actual situation is more prosaic and less polarized. Hawkins's papers do pass peer review and are published, in Nature no less. His ideas are improbable, but observationally testable. In due course, as more data on quasar fluctuations accumulate, his hypothesis is very likely to be disproved to the satisfaction of any sapient individual. If, on the other hand, his theory is observationally confirmed, then he will without doubt be acclaimed by the establishment, and I will eat this review. (It's brief, just in case.)

William H. Press is at Harvard College

Observatory, 60 Garden Street, Cambridge,

Massachusetts 02138, USA.

\section{Men behaving badly}

\section{Behavioral Genetics, third edition}

by Robert Plomin, John C. De Fries, Gerald E. McClearn and Michael Rutter

W. H. Freeman: 1997. Pp. 367. \$64.95, £26.95 Steven Rose

A basic textbook should act as an accessible, comprehensive and balanced introduction to the field of study, tailored to the needs of not only students but also their teachers, who are usually unlikely to be as well-versed in the topics as the textbook's authors. In innovatory or controversial areas, this imposes a severe responsibility on the authors. They may and indeed are likely to have a preferred take on the topics they cover. Nothing wrong with that: treated properly, it provides a refreshingly coherent pathway through often messy data.

But textbook authors who 'take a position' also have a responsibility to the students for whom their book will act as source material: they must make it clear where they are coming from, and at least acknowledge by reference the alternative positions they reject. And I know from experience that this is not always easy.

In few areas of modern science can this stricture be sharper than in the biological and especially the assumed genetic — causes of human behaviour. This is a field that has been vitriolic with methodological, interpretative and political disputes for its entire century-long history. The authors of Behavioral Genetics are no novices in this contentious area. Between them the first three have made substantial contributions to arguments that genetics is important in our understanding of individual differences in human behaviour. The fourth, a social psy- chiatrist, has over the past decades become increasingly interested in the role of genetics.

Their textbook aims to introduce the methods of quantitative and statistical population genetics, some of which the authors and their colleagues have pioneered, to students of psychology and psychiatry. They offer a quick whip through Mendel and single-gene disorders and 'exceptions' to Mendelism, and a beginner's guide to DNA, before embarking on their main task, staking the claim that quantitative genetics can explain the causes of important features of the human condition.

Unfortunately, the result is less a work of balanced scholarship than of passionate advocacy. Its aim, according to the preface, is to make the case that genetics is central to psychology. In attempting to achieve this goal, it is scrupulous with neither the data or their presentation. Few other textbooks are, for instance, illustrated by pictures of leading advocates in the field, supplemented by 'boxes' of uncritical hagiography, while ignoring - with the exception of the odd disparaging remark - equally distinguished psychiatrists, neuroscientists or geneticists who offer a less genetically determinist view of human behaviour.

Few competent textbooks of psychiatry describe such diagnostic categories as schizophrenia, cognitive, attention-deficit or personality disorders without allowing a shred of doubt as to the validity and stability of the categories offered. Some may, but none should, describe adoption studies as straightforward "experiments of nature" without any social reflection on the nonneutral significance of adoption for both the adoptee and his or her parents.

Indeed, the lack of appreciation of the social context, as if the vagaries of human behaviour were as simple to categorize as those of Drosophila in a choice maze, is one of the most depressing - and alarming, considering its intended audience of psychiatry students - features of this book. And few textbooks of genetics offer such adulatory accounts of the validity of quantitative trait locus analyses without at least reflecting on the problems of distinguishing biological reality from statistical artefact.

A textbook should encourage students to think for themselves, to develop a healthy respect for the science introduced and to cultivate a critical approach to assessing the theoretical and experimental validity of its claims. Otherwise students will be merely drilled into the latest fashionable theoretical mould.

In their zeal for pressing their own views, influential though they may be, the authors of this textbook have badly failed their basic educational responsibility.

Steven Rose is in the Brain and Behaviour Research Group, Open University, Milton Keynes MK7 6AA, UK. 